\title{
Síndrome de Hamman: Um Diagnóstico Diferencial de Dor Torácica
}

\section{Hamman Syndrome: A Differential Diagnosis of Chest Pain}

Sara Montezinho'*, João Cunha ${ }^{1}$ Gonçalo Sarmento ${ }^{1}$

\author{
*Autor Correspondente/Corresponding Author: \\ Sara Montezinho [saramontezinho@gmail.com] \\ Rua Dr. Cândido Pinho 5, 4520-211 Santa Maria da Feira, Portugal \\ ORCID iD: 0000-0003-0505-8925
}

PALAVRAS-CHAVE: Dor Torácica; Enfisema Mediastínico/diagnóstico; Enfisema Mediastínico/diagnóstico por imagem

KEYWORDS: Chest Pain; Mediastinal Emphysema/diagnosis; Mediastinal Emphysema/diagnostic imaging

\section{INTRODUÇÃO}

pneumomediastino espontâneo (também designado de síndrome de Hamman), é uma entidade rara, que resulta da rotura espontânea de alvéolos devido a um aumento rápido da pressão intratorácica. ${ }^{1}$ Pode ocorrer também na ausência de uma causa clara de aumento da pressão intratorácica, como tosse, exercício intenso ou vómito. ${ }^{2}$

\section{CASO CLÍNICO}

Jovem do sexo masculino, 21 anos, antecedentes de intolerância à lactose, sem medicação habitual, não fuma- dor e sem hábitos de exercício físico regular. Admitido no Serviço de Urgência por toracalgia anterior, constante, odinofagia e sensação de corpo estranho na garganta, com dois dias de evolução. Negava tosse, febre ou vómitos. Ao exame objetivo apresentava enfisema subcutâneo cervical à direita, eupneico com saturação periférica superior a 98\% (FiO 2 de 21\%) e auscultação pulmonar sem alterações. Estudo analítico normal, nomeadamente o doseamento do D-dímeros e marcadores de necrose miocárdica. Eletrocardiograma normal. Radiografia do tórax com evidência de pneumomediastino discreto (Fig. 1). Realizada tomografia computorizada (TC) torácica que tornou evidente acentuado enfisema subcutâneo cervical e torácico disperso e ligeiro pneumome- 


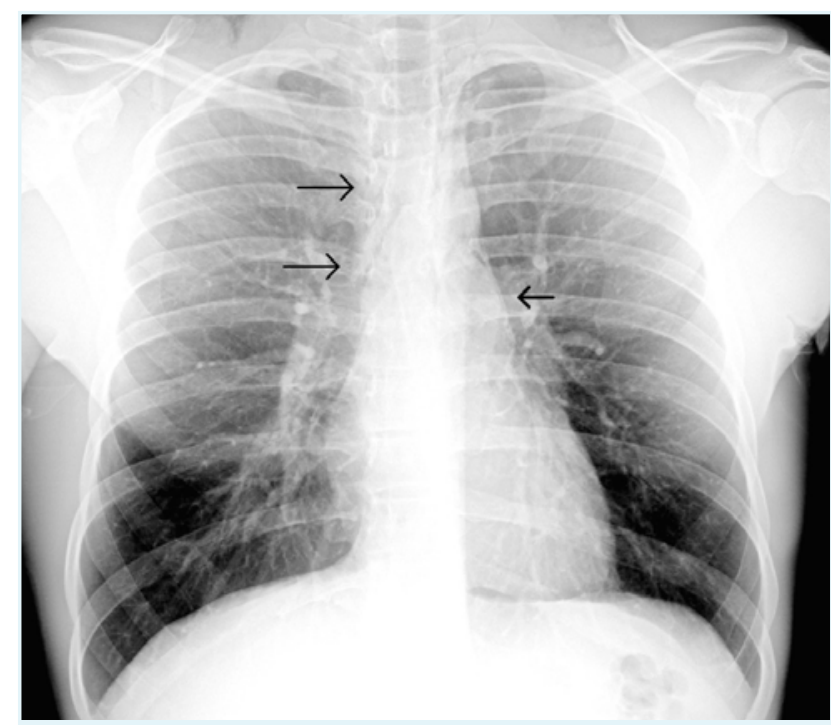

FIGURA 1. Radiografia do tórax, incidência ântero-posterior, com imagem subtil de pneumomediastino (setas).

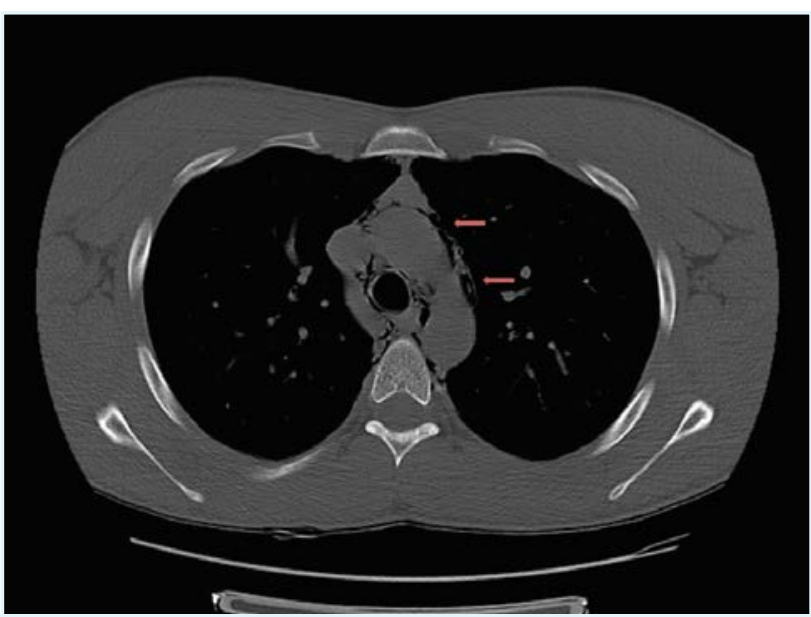

FIGURA 2. Tomografia computorizada, imagem em corte axial, com fina lâmina de ar em concordância com pneumomediastino (setas).

diastino (Fig. 2). O doente permaneceu internado sob oxigenoterapia e terapêutica analgésica, com regressão espontânea do pneumomediastino comprovada por repetição da imagem de TC.

\section{DISCUSSÃO}

pneumomediastino espontâneo é uma condição autolimitada, geralmente benigna, que responde bem ao tratamento conservador. ${ }^{3}$ Como descrito no caso apresentado, pode ocorrer sem qualquer fator precipitante e deve, por isso, ser considerado como diagnóstico diferencial de dor torácica. A radiografia do tórax exige algum grau de suspeição clínica e experiência, sendo a TC o exame mais adequado para o diagnóstico.

\section{RESPONSABILIDADES ÉTICAS}

CONFLITOS DE INTERESSE: Os autores declaram a inexistência de conflitos de interesse na realização do presente trabalho.

FONTES DE FINANCIAMENTO: Não existiram fontes externas de financiamento para a realização deste artigo.

CONFIDENCIALIDADE DOS DADOS: Os autores declaram ter seguido os protocolos da sua instituição acerca da publicação dos dados de doentes.

CONSENTIMENTO: Consentimento do doente para publicação obtido.

PROVENIÊNCIA E REVISÃO POR PARES: Não comissionado; revisão externa por pares.

\section{ETHICAL DISCLOSURES}

CONFLICTS OF INTEREST: The authors have no conflicts of interest to declare.

FINANCING SUPPORT: This work has not received any contribution, grant or scholarship.

CONFIDENTIALITY OF DATA: The authors declare that they have followed the protocols of their work center on the publication of data from patients.

PATIENT CONSENT: Consent for publication was obtained.

PROVENANCE AND PEER REVIEW: Not commissioned; externally peer reviewed.

\section{REFERÊNCIAS}

1. Dionísio P, Martins L, Moreira S, Manique A, Macedo R, Caeiro F, et al. Spontaneous pneumomediastinum: experience in 18 patients during the last 12 years. J Bras Pneumol. 2017;43:101-5.

2. Dajer-Fadel WL, Arguero-Sanchez R, Ibarra-Perez C, Navarro-Reynoso FP. Systematic review of spontaneous pneumomediastinum: a survey of 22 years' data. Asian Cardiovasc Thorac Ann. 2014;22:997-1002.

3. Kouritas VK, Papagiannopoulos K, Lazaridis G, Baka S, Mpoukovinas I, Karavasilis V, et al. Pneumomediastinum. J Thorac Dis. 2015;7(Suppl1):S44-S49. doi: 10.3978/j.issn.20721439.2015.01.11. 